\title{
Des Sciences Sociales à l'Anthropologie Cognitive
}

Les généalogies de la Cognition Située

From Social Science to Cognitive Anthropology: The "genealogies" of Situated

Cognition

\section{Benoit Grison}

\section{OpenEdition}

\section{Journals}

Édition électronique

URL : http://journals.openedition.org/activites/1230

DOI : $10.4000 /$ activites. 1230

ISSN : $1765-2723$

Éditeur

ARPACT - Association Recherches et Pratiques sur les ACTivités

\section{Référence électronique}

Benoit Grison, «Des Sciences Sociales à l'Anthropologie Cognitive », Activités [En ligne], 1-2 | octobre 2004, mis en ligne le 01 octobre 2004, consulté le 30 septembre 2020. URL : http:// journals.openedition.org/activites/1230; DOI : https://doi.org/10.4000/activites.1230

\section{c) (i) $\odot$}

Activités est mis à disposition selon les termes de la licence Creative Commons Attribution - Pas d'Utilisation Commerciale - Pas de Modification 4.0 International. 


\title{
Des Sciences Sociales à l'Anthropologie Cognitive. Les généalogies de la Cognition Située
}

\author{
Benoit Grison \\ Laboratoire Activité Motrice \& Conception Ergonomique (AMCO) \\ UFR STAPS d'Orléans \\ benoit.grison@univ-orleans.fr
}

RÉSUMÉ

Cet article propose une tentative d'《archéologie » de la cognition « située » et « distribuée », ensemble d'approches à l'interface des Sciences Cognitives et du constructivisme social. Trois courants historiques fondamentaux sont distingués, à l'origine, à travers diverses recombinaisons, de la majorité des formes d'action située actuelles : le courant de l' « action située » originelle, le courant de l' « apprentissage situé » et celui de la « cognition distribuée ».

MOTS-CLÉ

Activité située, cognition distribuée, cognition située, constructivisme social, microsociologie

\begin{abstract}
From Social Science to Cognitive Anthropology : The "genealogies" of Situated Cognition.

The "situated cognition" approach is not an unified paradigm; an historical analysis shows that it originated from the interaction of three distinct theoretical lineages: the first "situated action", the "situated learning" school and the "distributed cognition" perspective.
\end{abstract}

KEYWORDS

Distributed cognition, microsociology, situated activity, situated cognition, social constructivism

\section{1.- Introduction}

Depuis le milieu des années 1990, une communauté internationale de quelques centaines de chercheurs en Sciences Cognitives et Ergonomie se réclame d'une approche « située » et "distribuée » de la cognition. Il s'agirait d'envisager les processus cognitifs et l'activité comme indissociables d'une situation, dont les éléments physiques, artefactuels autant que sociaux, offrent des ressources signifiantes pour l'action des sujets (l'on relève ici l'influence de la théorie des affordances développée en Psychologie de la Perception par James Gibson, 1979). Par rapport au cadre théorique de la Psychologie Cognitive classique, l'accent est déplacé de l'étude de mécanismes internes vers celle de la psychological ecology, de l'« espace de vie » des acteurs - pour reprendre la terminologie de Kurt Lewin.

Cependant, malgré le succès croissant rencontré par le courant de la " cognition située », symbolisé par le ralliement spectaculaire d'un Jerome Bruner (cf. Bruner, 1990), celui-ci, plus qu'un paradigme unifié, ne forme encore, de l'aveu même d'un de ses promoteurs, qu'un « ensemble peu structuré de 
perspectives » (Resnick, 1996). L'on peut même se demander si la prise en compte tardive de son existence par le mainstream cognitiviste (le numéro spécial de 1993 de Cognitive Science) n'a pas conduit, à la suite de Vera et Simon (1993), à regrouper sous le label d' « action située » des approches hétérogènes parfois peu compatibles entre elles.

La notion même de contexte situationnel, qui relie les différents auteurs de l'action située, est mal débrouillée, d'autant plus qu'on est loin de disposer d'une théorie psychologique des situations! Néanmoins, le caractère « flou » et hétérogène de ce concept, comme souvent en science, ne l'empêche nullement d'être opératoire et de fonder des travaux intéressants. Nous verrons plus loin comment il fait sens pour des courants distincts. ${ }^{1}$

Une définition négative des caractéristiques communes des diverses recherches en action située peut d'ores et déjà être avancée :

- l'action située ne se confond nullement avec l'étude de l'activité en situation de travail, mais se caractérise bien par des présupposés théoriques forts, le plus souvent incompatibles avec le fonctionnalisme cognitiviste. Cela dit, si la perspective ergonomique dans son ensemble n'est pas « située » par nature, elle fournit néanmoins un terrain très adéquat pour tester les hypothèses de la cognition située. Bien plus, les chercheurs adhérant à une forme ou l'autre d'action située envisagent l'Ergonomie en tant que potentiellement porteuse de développements de connaissances scientifiques sur le domaine de l'activité humaine, voire même d'une théorie de l'activité. Ce faisant, ils récusent ainsi une dichotomie marquée entre « application » et recherche scientifique, et rejoignent les positions de pionniers tels que Ombredane ou Faverge qui concevaient l'analyse du travail comme une discipline «fondamentale» par ses contributions à l'étude de la cognition (cf. Richard, 1996). L'enjeu épistémologique pour l'Ergonomie est ici de taille.

- l'action située ne s'appuie pas exclusivement sur des méthodes d'observation «in vivo", mais également sur des techniques d'entretien, des simulations et des (quasi-) expérimentations.

- corrélativement, l'action située ne se réduit pas aux débats théoriques multiples autour de la « validité écologique » des situations de recueil de données, même si certains de ses pionniers ont fait avancer le questionnement à propos de cette notion. ${ }^{2}$

- anthropologie cognitive et cognition située ne sont pas entièrement synonymes : en effet, sans parler de l'« Ethnoscience» ou de l'« Anthropologie Structurale» des années 1960-1970, diverses anthropologies cognitives d'aujourd'hui, telles celles de Dan Sperber ou de Maurice Bloch, sont étrangères à l'approche située, alors qu'elles sont influencées pour partie par le cognitivisme.

Au-delà de la diversité des conceptions de l'action située incarnées par différents groupes de chercheurs, l'on retrouve quelques fondements théoriques récurrents : la référence à Vygotsky, aux microsociologies phénoménologique et compréhensive allemandes ; ainsi qu'à la postérité de l'Ecole de Chicago, marquée (sous l'influence du Pragmatisme) par une tradition de Psychologie Sociale interactionniste récusant le mentalisme. Cet enracinement commun explique l'intérêt porté par l'action située aux thèmes de la pensée pratique et de la « cognition incarnée ». L'on peut ainsi caractériser plus positivement les cognitions situées comme le produit de la rencontre entre la Psychologie Cognitive et le riche héritage historique du constructivisme social. Dès lors que des sociologues tels que Cicourel et Goffman s'intéressent à partir des années 1960 à la cognition et au

1. Pour des approches postérieures aux courants « classiques » de la cognition située abordés dans cet article, influencées par Varela (Theureau, 1992, par exemple) la question de la définition du contexte ne se pose plus, puisque ces modèles postulent une co-détermination de l'action et de la situation.

2. Sur ce point, voir Grison et Riff (2002) 
contexte microsocial de celle-ci, la voie est ouverte à un dialogue fructueux entre Sciences Cognitives et Sciences Sociales.

Pour mieux cerner les contours de ce champ de recherche mouvant, je me propose dans ce qui suit d'esquisser une généalogie des cognitions situées à travers la description brève de trois courants fondamentaux, qui, combinés à des degrés divers, me paraissent être à l'origine de la plupart des formes d'action située actuelles. Une telle approche "phylogénétique » peut permettre de circonscrire plus précisément ce qui fait la spécificité de l'approche située. Cette tentative de clarification historique visera également à dégager les aspects théoriques harmonisables entre les trois perspectives distinguées, et ceux qui ne le sont pas, ou alors au prix de syncrétismes problématiques. Enfin, je passerai pour conclure de la volonté de «neutralité » descriptive de l'Histoire des Idées à une approche critique explicite, esquissant le bilan épistémologique des apports des différentes cognitions situées aux Sciences Cognitives. Les trois courants dissociables sont les suivants :

1. Le courant de l'« action située » originelle : celui-ci s'enracine dans la tradition intellectuelle de l' «ethnométhodologie»d'Harold Garfinkel, sociologie novatrice privilégiant l'étude des « raisonnements sociologiques pratiques » des acteurs, ou ethnométhodes. L'ethnométhodologie conçoit la connaissance comme une construction locale (situated).

2. Le courant de l'« apprentissage situé » : il se situe dans le prolongement du programme de recherche développé depuis plus d'un siècle par la Psychologie Interculturelle (Cole, 1996) s'interrogeant sur l'existence d'invariants cognitifs face à la diversité des situations culturelles.

3. Le courant de la «cognition distribuée » : prend ses origines respectivement dans les problématiques de la Sociologie de la Connaissance et de l'école russe de Psychologie (Vygotsky et l'approche historico-culturelle de la cognition, la théorie de l'activité de Léontiev).

\section{2.- $L^{\prime}$ « action située » originelle ${ }^{3}$}

Dans son acception première, l'expression « action située » recouvrait un programme de recherche précis, mis en œuvre par des chercheurs formés à l'ethnométhodologie et à l'analyse conversationnelle, dont le chef de file fut Lucy Suchman $(1987,1988)$.

L'action située princeps vise à réévaluer la notion de planification, centrale dans les Sciences Cognitives classiques, en réintroduisant l'environnement de l'activité, absent de la vision « désincarnée » de la cognition véhiculée par le cognitivisme. Il s'agissait de réagir à une conception du planning élaborée sur le principe du GPS de l'I.A. (Newell, Shaw \& Simon, 1958), renvoyant à des stratégies de type « analyse des fins et des moyens », ou pensée comme séquence hiérarchisée d'opérations (Miller, Gallanter, \& Pribram, 1960).

En quête d'une théorie de l'action plutôt que d'un modèle du sujet connaissant, Suchman insiste avant tout sur la complexité changeante des situations, sur l'« autoorganisation émergente » de l'activité, sur la réactivité « opportuniste » des acteurs face aux contingences environnementales. Néanmoins, le fait qu'il n'y ait pas selon elle de prédictibilité possible des réactions des sujets, l'empêche de réhabiliter l'environnement quasi-déterministe du Behaviorisme dont elle se rapprocherait pourtant.

Je ne reviendrai pas ici sur les deux exemples concrets mis en avant par Suchman à l'appui de ses thèses sur la planification, l'étude de l'utilisation d'une photocopieuse et l'anecdote de la descente des rapides en canoë. L'essentiel est qu'elle récuse dans les deux cas l'idée de procédures de planifi-

3. A la suite de la publication du numéro de 1993 de Cognitive Science, l'habitude d'utiliser « action située » et « cognition située » comme des expressions synonymes s'est répandue, ce qui n'était pas le cas auparavant 
cation vues comme déterminants de l'action : selon elle, c'est bien plutôt l'action "locale», «située » qui est génératrice de plans. Non que les plans soient sans importance dans les actes quotidiens; au contraire : ils aident les sujets à faire sens de l'action. Il s'agira en fait de comprendre l'action, non de l'expliquer par des causes efficientes. De surcroît, l'on mettra désormais l'accent sur la question de l'improvisation, négligée par les cognitivistes : ainsi, lors de la descente en canoë, les difficultés rencontrées, pas toutes modélisables a priori, sont surmontées par le kayakiste en recourant à des skills " incorporées », issues d'expériences antérieures. Ce point de vue est une façon d'esquiver les apories sur lesquelles bute le « représentationnisme » strict (frame problem, paradoxe de l'« homonculus », ...). Mais la délibération, les processus de raisonnement de l'acteur passent alors au second plan des préoccupations de l'investigateur. A cet égard, les réflexions de Lucy Suchman sur le planning semblent en régression par rapport aux études pionnières de Gladwin $(1964 / 1974 ; 1970)$ sur les stratégies des navigateurs polynésiens Trukese (relayées plus tard par Hutchins) : cet anthropologue montrait que les marins des Carolines, partant comme leurs collègues européens d'une appréhension globale du problème (atteindre tel atoll), ne cherchaient pas à dégager un principe unificateur permettant de déduire l'ensemble des solutions particulières nécessitées au cours de l'action, mais s'appuyaient au fur et à mesure de son accomplissement sur des indices contextuels signifiants renvoyant à une pratique répétée (aspect des courants et îlots rencontrés, vents, voire même couleur de la mer...). Mais Gladwin n'envisageait pas l'action en contexte et l'action planifiée comme opposées, mais plutôt comme des composantes variables en degré des différents modes de navigation.

Quoi qu'il en soit, l'un des grands apports du groupe de Suchman a été de favoriser par ses travaux le passage de la notion de plan-programme à celle de plan-communication au sens où l'entend Phil Agre (Agre, \& Chapman, 1991). S'il y a planification, celle-ci est envisagée comme étant dynamique, le plan constituant un élément de l'action, sans la contrôler totalement : il devient en fait une ressource autorisant l'improvisation circonstancielle. Car, comme y insiste Kirsh (1990), la conception de l'action comme exclusivement «située » souffre de limitations intrinsèques : il apparaît nécessaire, quoi qu'on fasse, de coordonner des «contraintes locales» et des «contraintes globales » quand on passe d'un contexte à un autre.

Dans le prolongement des recherches de Suchman se situent également les premiers travaux de Microsociologie Cognitive de Bernard Conein et ses collaborateurs (Conein, \& Jacopin, 1993 \& 1994 ; Conein, 1998). Ces chercheurs sont marqués par le développement de l'« Artificial Life» dans le champ de la Robotique des années 1990 : celle-ci substitue alors avec succès des « robots réactifs ", « stimulus-réponse », aux anciens robots autonomes à représentations de connaissances, dotés de générateurs de plans (cf. Brooks, 1991). En conséquence, plutôt que de multiplier les processus internes hypothétiques, l'on tendra ici à se concentrer sur l'analyse du rôle que joue l'environnement dans les conduites intelligentes et sur les événements réels qui s'y produisent. Il s'agit clairement, comme le confirme la référence à l'œuvre de Mead (1934/1963), d'une reprise actualisée du behaviorisme social. ${ }^{4}$ Les objets d'étude privilégiés sont alors des dynamiques routinières complexes, ni règles internes, ni comportements adaptatifs « locaux » : l'on étudiera par exemple les rapports entre l'organisation concrète des objets de l'espace de travail, et l'exécution optimisée de recettes de cuisine, ou encore d'un travail de bureau (voir Fischler, \& Lahlou, 1995).

\section{3.- L'école de Michael COLE et le « situated learning "}

Des travaux interculturels ont été menés à partir des années 1960, d'abord sous l'impulsion du psychologue américain Michael Cole, sur l'« ethnomathématique » et l'influence de la scolarisation sur

4. Cette posture théorique ne correspond plus à l'état actuel de la pensée de Conein. 
les formes de raisonnement. Ces recherches de «psychologie culturelle» sur la cognition, surtout anglo-saxonnes, se sont développées en faisant abstraction de la conception alors dominante du cerveau comme «système de traitement de l'information». Elles ont conduit un groupe d'auteurs à définir l'importance capitale d'un «apprentissage situé » (situated learning), dont les produits seraient difficilement transférables à la résolution de problèmes dans d'autres situations.

Ainsi, Michael Cole, John Gay, et al. (1971) montrent que les enfants Kpelle du Libéria sont capables de performances arithmétiques étonnantes dans le cadre de la vie courante (les Kpelle sont les « maitres de la mesure du riz » disent ces chercheurs), mais peuvent avoir des difficultés à solutionner un problème scolaire de même niveau. Jean Lave a étudié pareillement des tailleurs libériens de l'ethnie Vai, répartis en 4 groupes : scolarisés / non scolarisés * experts / novices (cf. Lave, 1988). Les sujets sont confrontés à deux types de problèmes mathématiques de difficulté comparable (des calculs de proportions), l'un renvoyant à leur pratique professionnelle, l'autre non. Il y aurait une différence de performance positive de la part des individus scolarisés sur les problèmes ne faisant pas référence à la pratique (mais les données statistiques, incomplètes, ne permettent pas une interprétation univoque). De même, les enfants, vendeurs de rues de Recife étudiés par Carraher, Carraher, \& Schliemann, (1985) montrent des différences dans leurs stratégies arithmétiques professionnelles et celles déployées à l'école : celles de travail sont moins générales. Ce sont des heuristiques personnelles parfaitement adéquates à leur objet, à opposer à l'algorithmique scolaire.

Le groupe de Cole a par la suite transposé sa démarche, l'appliquant aux situations de la Psychologie Scolaire et de l'Ergonomie des sociétés industrielles. Lave et ses étudiants se sont penchés sur l'arithmétique quotidienne de ménagères faisant leurs courses, en train d'évaluer les prix les plus avantageux, sur les estimations empiriques de quantités de nourriture chez les weight watchers (Lave, 1988) : là encore, un décalage avec les performances de calcul de type scolaire des mêmes sujets, moindres, a été retrouvé. Scribner, quant à elle, s'est intéressée aux méthodes d'inventaire des laitiers d'une compagnie américaine de distribution en fonction du niveau d'expertise, Beach à l'activité de barman-experts (cf. Scribner, 1984).

Peu à peu, les recherches des tenants du situated learning les ont amené à relativiser le caractère « extraterritorial » des situations formées par l'école ou le laboratoire de Psychologie, jusqu'à envisager celles-ci comme des situations culturelles parmi d'autres. Les processus cognitifs sont vus comme partiellement liés à ces divers contextes de développement. Lave scinde le concept de « situation » en deux, distinguant l'arena, cadre institutionnel relativement stabilisé (le supermarché des ménagères, par exemple) du setting, couplage interactif de l'individu avec cette arena.

Ce dernier auteur a durci les perspectives issues de l'« apprentissage situé », pour finir par produire une version radicale de ce courant, proche de l'«action située» selon Suchman : la cognition se construirait entièrement dans l'action, les motifs de l'acteur n'étant que reconstructions rétrospectives. Qui plus est, pour Lave, « localiste » jusqu'au bout, l'existence d'une possibilité de transfert de compétences expertes d'un contexte culturel à un autre est douteuse. Ce point de vue n'est pas soutenable : des études récentes montrent bien qu'un transfert au moins partiel de procédures de raisonnement entre deux domaines de connaissances limitrophes, sans « régression » au niveau du novice, peut exister (voir e.a. Schraagen, 1993 ; Broyon, 2001).

Un apport important du courant de Michael Cole est l'insistance sur le rôle des artefacts dans l'activité : ceux-ci (que cela soit des récipients alimentaires pour le weight watcher, ou des cailloux pour l'enfant Kpelle hors des murs de l'école) peuvent être amenés ainsi à servir de dispositifs externes de calcul. Bien plus, à travers la réévaluation de l'œuvre de Vygotsky et son travail avec Jack Goody sur les conséquences cognitives de l'alphabétisation en Afrique de l'Ouest, Cole est amené à se pencher sur le livre comme outil de pensée réorganisateur de la cognition (Scribner, \& Cole, 1981). 


\section{4.- La Cognition Distribuée}

La « cognition distribuée » est un programme de recherche qui bénéficie maintenant d'une visibilité notable en Ergonomie, en Psychologie du Travail et dans le domaine de l'Éducation (cf. e.a. Salomon, 1993) : à cet égard, le livre de Hutchins, Cognition in the Wild (1995) a constitué un tournant. Il n'en reste pas moins que l'existence de ce courant est déjà ancienne, et remonte en fait à plusieurs décennies. Son avénement a été préparé à la fois par la "psychologie anthropologique » issue de Wundt, qui concevait les processus supérieurs comme étant d'origine culturelle (cf. Wundt, 1916 ; Roberts, 1964), et par la sociologie phénoménologique d'Alfred Schütz.

Le représentant le plus notable de la première tendance est bien entendu le psychologue russe Vygotsky (cf. Riviere, 1990). Vygotsky, et à sa suite Léontiev, qui intègre les objets dans sa vision du système complexe formé par l'activité psychologique, distinguent deux types d'instruments : les instruments techniques sensu stricto, transformateurs des objets eux-mêmes, de l'environnement, et les instruments psychologiques (l'écriture, les algorithmes de calcul, les abaques, les cartes,...), réorganisant la cognition individuelle. Cette dernière notion a été retrouvée par Latour (1985) avec ses « inscriptions » et même par Simon qui a insisté sur le rôle des diagrammes dans le raisonnement des scientifiques (Larkin, \& Simon, 1987). Elle est désormais plutôt désignée sous le terme d' « artefact cognitif » (Norman, 1993).

Quant à Schütz, il fut le premier à développer explicitement dès les années 1940 un modèle du social mettant l'accent sur la « distribution de la connaissance » - pour reprendre ses propres termes - entre individus et objets culturels : ces stocks de connaissances « socialement dérivées », intersubjectives, sont mobilisés par les individus dans des contextes situationnels précis (cf. Schütz, 1964). ${ }^{5}$ Pour le sociologue austro-américain, l'étude de cette distribution cognitive devrait être l'objectif même d'une sociologie de la connaissance conséquente. Schütz, au contraire des psychologues vygotskyiens, s'est peu intéressé aux « systèmes d'amplification cognitive » - l'exception étant quelques pages qu'il a consacrées aux partitions musicales, mais leur point de départ lui avait été suggéré par un texte d'Halbwachs (1939).

Dans la version actuelle de la cognition distribuée, dont le développement a été catalysé par l'apparition des simulations d'Intelligence Artificielle Distribuée (Chandrasekaran, 1981; Ferber, 1995 ; Doran, Troitzsch, Mueller, \& Gilbert, 1996), la culture est conçue comme système « discrétionnaire », de collecte, stockage, traitement et récupération des connaissances, formé par le réseau des systèmes cognitifs individuels et des artefacts. Pour l'investigateur, les systèmes de cognition collective constituent alors les unités d'analyse, dont il s'agit de comprendre le fonctionnement d'ensemble (l'exemple célèbre du cockpit de l'avion de ligne étudié par Hutchins). Contrairement à ce que laisse entendre Nardi (1996), le sujet cognitif n'est pas nié à travers cette approche : simplement, au niveau d'analyse où elle se place, la question subjectale n'a pas de pertinence. $^{6}$

Le rapport qu'entretient la cognition distribuée avec le cognitivisme est ambigu, comme en témoigne le vocabulaire «computationnel» employé par Hutchins et d'autres auteurs : «flux d'informations », " représentations de connaissances », ... Il y a pourtant incompatibilité entre les conceptions de la cognition constructivistes et « historico-culturelles» à l'origine de la distributed cognition d'une part, et le fonctionnalisme cognitiviste d'autre part.

5. Que la cognition distribuée ait été longtemps dénommée également « cognition socialement partagée » trahit bien son origine phénoménologique partielle ( $\mathrm{cf}$. le « monde partagé par tous » des philosophes phénoménologues).

6. Par contre, la Théorie des Acteurs-Réseaux et l'« interobjectivité » de Bruno Latour (cf. Courtial, 1994 ; Latour, 1994), qui symétrisent réellement les statuts des non-humains et des humains, ne sont pas conciliables avec les fondements théoriques initiaux de la cognition distribuée, notamment la sémiotique subjectale de Bakhtine et Vygotsky, que ces derniers avaient hérité de leur maître, le phénoménologue et linguiste Gustav Shpet (voir à ce sujet Zinchenko, 2002). 


\section{5.- Gains et / ou régressions épistémologiques ?}

Le paradigme cognitiviste avait pour corrélat un objectivisme strict, supposant l'établissement de correspondances univoques entre les représentations et l'univers extérieur. Ce faisant, il échouait à rendre compte d'une caractéristique essentielle du système cognitif humain : celui-ci est fondamentalement un « système interprétant», donneur de sens. Avec l'action située, l'on passe du traitement d'information à la construction culturelle de significations, et dans le même temps, d'une approche explicative à une approche compréhensive des processus. La cognition de l'être humain y est vue comme incarnée et historiquement construite : les différentes formes de cognition située peuvent donc s'harmoniser avec le point de vue des neurobiologistes, qui pour la plupart rejettent l'idée du cerveau comme Machine de Turing (Edelman, 1992 ; Prochiantz, 1997).

Pour autant, refuser d'adhérer à un réalisme naïf n'implique nullement d'embrasser les formes radicales de relativisme épistémologique... C'est pourtant le choix qu'ont fait les tenants de «versions fortes » de l'action située telles Lave et Suchman : le «localisme» de ces auteurs les dissuade d'accorder aux vérités scientifiques un caractère universel possible; ce faisant, ce «nihilisme épistémologique» les conduit à se rapprocher de diverses formes de sociologie des sciences ultrarelativistes (Latour ou encore le «programme fort» des sociologues anglais). Avec de tels « constructivismes durs », où les sciences (fussent-elles cognitives) ne sont considérées que comme des constructions culturelles parmi d'autres, l'on est loin de l'attitude épistémologique d'un Harold Garfinkel. En effet, ce dernier a toujours sauvegardé un réalisme minimal, considérant, à l'instar de Thomas Huxley naguère, la science comme une forme élaborée de sens commun. Le lien entre cognition située et relativisme épistémologique radical est tout sauf nécessaire.

\section{BIBLIOGRAPHIE}

Agre, P.E., \& Chapman, D. (1991). What are plans for? In P. Maes (Ed.), Designing Autonomous Agents, Cambridge, MA: MIT Press, pp. 17-34

Brooks, R. (1991). New approaches to Robotics. Science, 253, 1227-1232.

Broyon, M.A. (2001). Métacognition et cultures. In Actes $8^{\text {ème }}$ Congrès de l'Association pour la Recherche InterCulturelle (ARIC), 24-28 septembre, Université de Genève.

Bruner, J. (1990). Acts of Meaning. Cambridge, MA: Harvard University Press.

Carraher, T.N., Carraher, D.W., \& Schliemann, A.D. (1985). Mathematics in the streets and in the schools. British Journal of Developmental Psychology, 3, 21-29.

Chandrasekaran, B. (1981). Natural and social system metaphors for distributed problem solving : introduction to the issue. IEEE Transactions on Systems, Man and Cybernetics SMC, 11, 1-5.

Cole, M. (1996). Cultural Psychology: A Once and Future Discipline. Cambridge, MA: Harvard University Press.

Cole, M., Gay, J., Glick, J.A., \& Sharp, D.W. (1971). The cultural context of learning and thinking. New York: Basic Books.

Conein, B. (1998). La notion de routine : problèmes de définition. Sociologie du Travail, 40 (4), 479-489.

Conein, B., \& Jacopin, E. (1993). Les objets dans l'espace : la planification dans l'action. In B. Conein, N. Dodier et al. (Eds), Les objets dans l'action. Paris: EHESS, 59-84.

Conein, B., \& Jacopin, E. (1994). Action située et cognition : le savoir en place. Sociologie du Travail, 36 (4), 475-500.

Courtial, J.P. (1994). Science Cognitive et Sociologie des Sciences. Paris: PUF.

Doran, J., Troitzsch, K.G., Mueller, U., \& Gilbert, G.N. (1996). Social Science Microsimulation. Berlin: Springer.

Edelman, G. (1992). Biologie de la conscience. Paris: Odile Jacob.

Ferber, J., (1995). Les systèmes multi-agents : vers une intelligence collective. Paris: Interéditions. 
Fischler, C., \& Lahlou, S. (1995). Dossiers, piles d'attente et corbeilles. La digestion quotidienne de l'information dans l'entreprise. Rapport EDF-DER HN 51/95/017, octobre.

Gibson, J.J. (1979). The ecological approach to visual perception. Boston: Houghton-Mifflin.

Gladwin, T. (1970). East is a Big Bird. Cambridge, MA: Harvard University Press.

Gladwin, T. (1974). Culture and logical process (1964). In J.W. Berry, \& P. Dasen (Eds.), Culture and cognition - readings in cross-cultural Psychology. London: Methuen, 27-37.

Grison, B., \& Riff, J. (2002). Validité écologique et situations d'étude privilégiées : de la psychologie expérimentale à l'anthropologie cognitive située. In Actes $4^{\text {èmes }}$ Journées d'Etudes de l'Association ACT'ING, 'Objets théoriques, objets de conception, objets d'analyse et situations d'étude privilégiées', 6-7 juin, Domaine de Chalès, Sologne.

Halbwachs, M. (1939). La mémoire collective chez les musiciens. Revue Philosophique, mars-avril, n 3-4, 136-165.

Hutchins, E. (1995). Cognition in the Wild. Cambridge, MA: MIT Press.

Kirsh , D. (1990). Préparation et improvisation. Réseaux, n 43, 111-120.

Larkin, J., \& Simon, H.A. (1987). Why a diagram is (sometimes) worth ten thousand words. Cognitive Science, 11, 65-99.

Latour, B. (1985). Les « vues » de l'esprit : une introduction à l'anthropologie des sciences et des techniques. Culture-Technique, ${ }^{\circ} 14$, juin, 5-29.

Latour, B. (1994). Une sociologie sans objet? Note théorique sur l'interobjectivité. Sociologie du Travail, 36 (4), 587-607.

Lave, J. (1988). Arithmetics in Practice. Cambridge, MA: Harvard University Press.

Léontiev, A.N. (1984). Activité, conscience, personnalité (1975). Paris: Éditions Sociales.

Mead, G.H. (1963). L'esprit, le soi, la société (1934). Paris: PUF.

Miller, G., Gallanter, E., \& Pribram, K. (1960). Plans and the structure of behaviour. New York: Holt, Rinehart \& Winston.

Nardi, B. (1996). Studying context. In B. Nardi (Ed.), Context and Consciousness. Cambridge, MA: MIT Press, 69-102.

Newell, A., Shaw, J.C., \& Simon, H.A. (1958). Elements of a theory of human problem-solving. Psychological Review, 65, 151-166.

Norman, D.A. (1993). Les Artefacts Cognitifs. In B. Conein, N. Dodier et al. (Eds.), Les objets dans l'action. Paris: EHESS, 15-34.

Prochiantz, A. (1997) Les Anatomies de la Pensée. Paris: Odile Jacob.

Resnick, L.B. (1996). Le rationalisme situé. Perspectives, 26, 1, mars.

Richard, J.F. (1996). Les différentes approches de l'analyse des compétences cognitives. Psychologie Française, 41 (1), 3-8.

Riviere, A. (1990). La Psychologie de Vygotsky. Liège: Mardaga.

Roberts, J. (1964). The self-management of cultures. In W. Goodenough (Ed.), Explorations in Cultural Anthropology. New York: McGraw Hill, pp. 433-454

Salomon, G. (1993). Distributed Cognitions. Cambridge: Cambridge University Press.

Schraagen, J.M. (1993) How experts solve a novel problem in experimental design. Cognitive Science, 17, 285-309.

Schütz, A. (1964). Collected Papers (tomes 1 \& 2). The Hague: Nijhoff.

Scribner, S. (1984). Cognitive studies of work. The Quarterly Newsletter of the Laboratory of Comparative Human Cognition, 6, 1-2, January-April, 1-49.

Scribner, S., \& Cole, M. (1981). The Psychology of Literacy. Cambridge, MA: Harvard University Press.

Suchman, L. (1987). Plans and Situated Actions: the problem of human-machine communication. Cambridge: Cambridge University Press. 
Suchman, L. (1988). Representing practice in Cognitive Sciences. Human Studies, 11 (2-3), 305-325.

Theureau, J. (1992). Le cours d'action : analyse sémio-logique. Berne: Peter Lang.

Vera, A.H., Simon, H.A. (1993). Situated Action: a symbolic interpretation. Cognitive Science, 17, 7-48.

Wundt, W. M. (1916). Elements of Folk Psychology : outlines of a psychological history of the development of mankind. New York: Macmillan.

Zinchenko, V. (2002). Contributions of Shpet's thinking to Vygotskian elaborations. In Dealing with Diversity, Book of abstracts of the $5^{\text {th }}$ Congress of the International Society for Cultural Research and Activity Theory (ISCRAT), 18-22 June, Amsterdam (p.239).

\section{RÉFÉRENCEMENT}

Grison, B. (2004). Des Sciences Sociales à l'Anthropologie Cognitive. Les généalogies de la Cognition Située, @ctivités, 1 (2), 26-34. http://www.activites.org/v1n2/grison.pdf 